\title{
Avaliação dos efeitos da natação como reabilitação pós-operatória na regeneração nervosa de ratos da linhagem Wistar submetidos a enxerto de nervos autólogos após lesão do nervo ciático*
}

\section{Assessment of the Effects of Swimming as a Postoperative Rehabilitation on Nerve Regeneration of Wistar Rats Submitted to Grafting of Autologous Nerves after Injury to the Sciatic Nerve}

\author{
Igor Rabelo de Sales Andrade ${ }^{1}$ Luis Renato Nakachima ${ }^{1} \quad$ Marcela Fernandes ${ }^{1}$ \\ Carlos Henrique Fernandes ${ }^{1}$ João Baptista Gomes dos Santos ${ }^{1}$ Sandra Gomes Valente ${ }^{1}$
${ }^{1}$ Departamento de Ortopedia e Traumatologia, Universidade Federal de São Paulo, São Paulo, SP, Brasil
Endereço para correspondência Igor Rabelo de Sales Andrade, R. Borges Lagoa, 570 - Vila Clementino, São Paulo, SP, 04038-000, Brasil Rev Bras Ortop 2020;55(3):323-328. (e-mail: igorrsa@hotmail.com).

\begin{abstract}
Resumo
Objetivo Avaliar os efeitos da natação na regeneração nervosa após a lesão do nervo ciático em ratos Wistar.

Métodos Um total de 30 ratos Wistar foram divididos em 3 grupos: grupo Sham + Nat: animais que não foram submetidos à cirurgia de enxerto e foram submetidos à natação $(n=10)$; grupo Enxerto: animais que foram submetidos à cirurgia de enxerto autólogo de nervo ciático $(n=10)$; e grupo Enx + Nat: animais submetidos à cirurgia de enxerto autólogo de nervo ciático e à natação $(n=10)$. Os resultados foram analisados pelo software GraphPad Prism 5.0 (GraphPad Software, San Diego, CA, EUA).

Resultados Na primeira avaliação, todos os valores do índice funcional do ciático (IFC) foram semelhantes $(p=0.609$ ). Após 30 dias do procedimento cirúrgico, foram observadas diferenças entre todas as comparações: Sham + Nat $(-34,64 \pm 13,89)$ versus Enxerto $(-145,9 \pm 26,06)$, grupos Sham + Nat versus Enx + Nat $(-89,40 \pm 7,501)$, grupos Enxerto $(-145,9 \pm 26,06)$ versus Enx + Nat $(-89,40 \pm 7,501)$. Nas medidas (60 e 90 dias), não

Palavras-chave

- traumatismos dos nervos periféricos

- regeneração nervosa

- natação

- nervo ciático houve diferença estatística entre os grupos Enxerto e Enx + Nat, com valores significativamente menores em relação ao grupo controle $(p<0,001)$. O número de motoneurônios apresentou diferenças nas comparações entre os grupos Sham + Nat e Enxerto $(647,1 \pm 16,42$ versus $563,4 \pm 8,07 ; p<0,05)$ e Sham + Nat e Enx + Nat $(647,1 \pm 16,42$ versus 558,8 $\pm 14,79 ; p<0,05)$, não havendo diferença entre os grupos Enxerto $\mathrm{e}$ Enx + Nat.
\end{abstract}

Trabalho desenvolvido no Departamento de Ortopedia e Traumatologia da Universidade Federal de São Paulo, São Paulo, SP, Brasil recebido

13 de Fevereiro de 2019

aceito

13 de Maio de 2019
DOI https://doi.org/

10.1055/s-0039-1692711. ISSN 0102-3616.
Copyright $\odot 2020$ by Sociedade Brasileira License terms de Ortopedia e Traumatologia. Published by Thieme Revnter Publicações Ltda, Rio de Janeiro, Brazil 


\begin{abstract}
Keywords

- peripheral nerve injury

- nerve regeneration

- swimming

- sciatic nerve

Objective To evaluate the effects of swimming on nerve regeneration after sciatic nerve injury in Wistar rats.

Methods A total of 30 Wistar rats was divided into 3 groups: Sham + Nat group animals that were not submitted to graft surgery and were submitted to swimming $(n=10)$; Graft group: animals submitted to autologous sciatic nerve graft $(n=10)$; and Graft + Nat group: animals submitted to autologous sciatic nerve graft surgery and to swimming $(n=10)$. The results were analyzed on the software (GraphPad Software, San Diego, CA, USA).

Results In the first evaluation, all sciatic functional index (SFI) values were similar $(p=0.609)$. Thirty days after the surgical procedure, we observed differences between all the comparisons: Sham + Nat $(-34.64 \pm 13.89)$ versus Graft $(-145.9 \pm 26.06)$; Sham + Nat versus Graft + Nat $(-89.40 \pm 7.501)$; Graft $(-145.9 \pm 26.06)$ versus Graft + Nat $(-89.40 \pm 7.501)$. In the measurements (60 and 90 days), there was no statistical difference between the Graft and Graft + Nat groups, with significantly lower values in relation to the control group $(p<0.001)$. The number of motor neurons presented differences in the comparisons between the Sham + Nat and Graft groups (647.1 \pm 16.42 versus $563.4 \pm 8.07$; $p<0.05)$, and between the Sham + Nat and Graft + Nat groups (647.1 \pm 16.42 versus $558.8 \pm 14.79 ; p<0.05)$. There was no difference between the Graft and Graft + Nat groups. Conclusion Animals submitted to the swimming protocol after the sciatic nerve grafting procedure did not present differences in the SFI values and motor neuron numbers when compared to the control group. Therefore, this type of protocol is not efficient for the rehabilitation of peripheral nerve lesions that require grafting. Therefore, further studies are needed.
\end{abstract}

Conclusão Os animais submetidos ao protocolo de natação após o procedimento de enxerto do nervo ciático não apresentaram diferenças nos valores de IFC e nos números de motoneurônios quando comparados com grupo controle. Portanto, este tipo de protocolo não é eficiente para reabilitação de lesões nervosas periféricas que necessitam de enxerto, sendo necessários novos estudos.

\section{Introdução}

As lesões dos nervos periféricos são frequentes na prática clínica e representam um problema de saúde capaz de gerar incapacidade na população. ${ }^{1}$ Os fatores etiológicos mais associados às lesões incluem: acidente automobilístico, lesão penetrante, e lesões relacionadas ao esporte. ${ }^{2}$

Morfologicamente, ocorre um aumento no tecido conjuntivo intramuscular e atrofia muscular evidenciada pela diminuição na área da secção transversal das fibras musculares. ${ }^{3}$ Tais alterações causam dor e sensibilidade ao longo da fibra nervosa, ${ }^{4}$ que podem resultar em limitações aos pacientes.

Apesar dos nervos periféricos possuírem a capacidade de regeneração, ${ }^{5}$ a recuperação é criticamente dependente do tratamento pós-lesão. ${ }^{1}$ Tratamentos não cirúrgicos, como o exercício físico, podem atuar na regeneração nervosa periférica. ${ }^{4}$

O exercício físico executado no ambiente aquático induz efeitos fisiológicos que proporcionam benefícios aos sistemas cardiovascular, esquelético, muscular e nervoso que aumentam o processo de reparação tecidual. ${ }^{6}$ A natação auxilia na regeneração do nervo ciático em coelhos, ${ }^{7}$ peixes ${ }^{8}$ e ratos. ${ }^{9-11}$ Esta parece atuar na remoção da mielina degenerada e na sua síntese na fase regenerativa, ${ }^{7}$ acelerando a recuperação nervosa. ${ }^{10,11}$
A imersão em ambientes aquáticos gera efeitos fisiológicos, como a elevação geral na temperatura corporal. À medida que a pele se torna aquecida, os vasos sanguíneos superficiais dilatam, o suprimento sanguíneo periférico é aumentado, e a frequência cardíaca eleva. O calor advindo da água reduz a sensibilidade das terminações nervosas sensitivas, auxiliando no alivio da dor e na redução do espasmo muscular. ${ }^{12,13}$ Embora haja estudos que tenham mostrado que a natação não promove benefícios na recuperação sensorimotora de ratos após a lesão do nervo ciático. $^{14,15}$

Os efeitos da natação na regeneração nervosa são conflitantes, pois alguns autores relataram que os exercícios forçados podem ter efeitos prejudiciais, especialmente no restabelecimento da função muscular. ${ }^{16,17} \mathrm{O}$ estresse induzido pelo treinamento físico poderia impedir a recuperação funcional após lesão nervosa.

Devido às controvérsias científicas dos benefícios da natação, são necessários estudos que mostrem os efeitos desta atividade física na regeneração do nervo ciático. Neste contexto, o presente estudo tem como objetivo avaliar os efeitos da natação na regeneração nervosa após lesão do nervo ciático em ratos da linhagem Wistar. 


\section{Materiais e Métodos}

\section{Casuística}

Foram utilizados 30 ratos machos da linhagem Wistar provenientes do Centro de Desenvolvimento de Modelos Experimentais, com idade de entre 7 e 8 semanas, pesando em média $250 \mathrm{~g}$. Os animais foram mantidos em condições controladas de iluminação (ciclo claro/escuro de 12 horas), temperatura controlada $\left(21 \pm 2^{\circ} \mathrm{C}\right)$ e livre acesso à água e ração. Todos os animais foram mantidos em biotério até o momento da eutanásia.

O presente trabalho seguiu as normas da legislação vigente para experimentação animal, de acordo com as com as normas nacionais e a legislação internacional (Diretrizes do Colégio Brasileiro de Experimentação Animal, COBEA; Guia dos Institutos Nacionais da Saúde [NIH, na sigla em inglês] para Cuidados e Uso de animais de laboratório). 0 presente trabalho foi aprovado pela Comissão de Ética no Uso de Animais (CEUA), sob o número 5060051217).

\section{Definição dos grupos de estudos}

Os animais foram divididos aleatoriamente em três grupos:

- SHAM + NATAÇÃO (Sham + Nat) - Animais que foram submetidos ao procedimento cirúrgico, sem retirada de enxerto, e foram submetidos à natação $(n=10)$;

- ENXERTO (Enxerto) - Animais que foram submetidos à cirurgia de enxerto autólogo de nervo ciático $(n=10)$;

- ENXERTO + NATAÇÃO (Enx + Nat) - Animais submetidos à cirurgia de enxerto autólogo de nervo ciático e à natação $(n=10)$.

\section{Procedimento cirúrgico}

No grupo Sham + Nat, o nervo ciático direito foi exposto por 10 minutos, e a camada muscular foi suturada com nylon monofilamentar 40 (Monofilamento preto NY44CT30, Tecnofil, Goiânia, GO, Brasil). Nos outros grupos, o nervo ciático foi cortado e um segmento de $8 \mathrm{~mm}$ foi ressecado, deixando um coto distal $\sim$ $3 \mathrm{~mm}$ antes da ramificação nervosa, como descrito por Fernandes et al. ${ }^{18} \mathrm{O}$ segmento do nervo, agora considerado enxerto de nervo, foi invertido e suturado com 2 pontos de fio monofilamentar 10-0 (Microcirurgia preto N-10005, Techsuture, Bauru, SP, Brasil). ${ }^{19} \mathrm{~A}$ pele e o músculo foram suturados com fio monofilamentar de nylon 4-0 (Monofilamento preto NY44CT30, Tecnofil, Goiânia, GO, Brasil), e a operação foi encerrada.

\section{Pós-operatório}

Após a cirurgia, os ratos foram mantidos em gaiolas, submetidos à dieta padrão do biotério, água ad libitum, e a um ciclo claro/escuro de 12 horas. 0 tratamento imediato foi realizado com a administração de cloridrato de tramadol ( $5 \mathrm{mg} / \mathrm{kg}$, intraperitoneal). Em seguida, foi disponibilizado o tratamento com paracetamol e cetoprofeno por via oral adotando a dose preconizada para ratos de $1,5 \mathrm{mg} / \mathrm{ml}$ e $5 \mathrm{mg} / \mathrm{kg}$, respectivamente, a cada 24 horas, durante $\geq 5$ dias, se houvesse sinais de dor.

\section{Avaliação da marcha}

A avaliação da marcha foi realizada antes do procedimento cirúrgico (tempo 0) e após 30, 60 e 90 dias. Os animais foram avaliados através do sistema CatWalk XT (Noldus Information Technology Inc., Leesburg, VA, EUA), que analisa pegadas através de uma passarela transparente acoplada a um sistema de computador. Estas pegadas foram utilizadas para calcular o índice funcional do ciático (IFC). ${ }^{19}$

\section{Protocolo da natação}

Os ratos dos grupos Sham + Nat e Enx + Nat foram colocados em uma piscina de $55 \mathrm{~cm}$ de altura, a mesma foi preenchida com 150 litros de água a uma temperatura de $30 \pm 2^{\circ} \mathrm{C}$, e os ratos foram induzidos a nadar. Antes do procedimento cirúrgico, os animais passaram por um período de adaptação progressiva durante 5 dias, iniciando o treinamento com 20 minutos até atingir 1 hora de natação. 0 exercício de natação foi iniciado no $14^{\circ}$ dia após a cirurgia, com duração de 2 semanas, no qual os animais nadaram 30 minutos por 5 vezes na semana. Após esta etapa, os animais executaram a atividade de natação por 9 semanas seguindo o cronograma de 30 minutos por 3 vezes na semana. ${ }^{9}$

\section{Cirurgia para marcação neuronal}

A marcação neuronal foi realizada com o marcador neuronal retrógrado Fluoro-Gold (Fluorochrome, LLC, Denver, CO, USA), em uma concentração de $3 \%$, no mesmo procedimento, através da exposição do coto proximal, distal à $2^{\underline{a}}$ sutura do nervo durante 90 minutos, para posterior contagem dos neurônios no corno anterior da medula. ${ }^{20}$

Perfusão transcardíaca e extração da medula espinhal A perfusão transcardíaca foi realizada 48 horas após a marcação neuronal, através de abertura da caixa torácica e exposição do coração. $\mathrm{O}$ ventrículo esquerdo foi puncionado com agulha e as soluções infundidas foram drenadas através de uma secção do átrio direito. Durante a perfusão, foram utilizadas as soluções tampões fosfato $0,2 \mathrm{M}$, paraformaldeído $4 \%$ e sacarose $10 \%$, em pH fisiológico. ${ }^{20}$

Imediatamente após a perfusão, foi realizada a laminectomia e um segmento da medula espinhal foi removido, usando um microscópio cirúrgico (MCT MU-M 19, DFVasconcelos, São Paulo, SP, Brasil). As raízes de L3 e S1 foram identificadas na origem e seccionadas transversalmente de modo a manter os segmentos L4, L5 e L6 intactos. Os segmentos da medula espinhal foram crioprotegidos em sacarose $20 \%$ e seccionados em criostato em uma espessura $40 \mu \mathrm{m}$. As fatias foram montadas em lâminas de vidro e analisadas por microscopia de fluorescência. ${ }^{18}$

\section{Análise histológica do segmento de medula}

Para a análise histológica, foi feito um estudo quantitativo de neurônios marcados pelo marcador neuronal retrógrado Fluoro-Gold no corno anterior da medula. Utilizando os aumentos de 25, 50, 100 e 400 vezes do microscópio de fluorescência ZEISS Axiolab (Carl Zeiss AG, Oberkochen, Alemanha), todas as secções foram examinadas e os motoneurônios marcados foram computados. Todas as análises foram realizadas pelo mesmo examinador. A análise histológica foi realizada aleatoriamente na medula de cinco animais de cada grupo. Nesta, foram consideradas apenas as células fortemente 
positivas para Fluoro-Gold, sendo utilizado o critério de correção de Abercrombie (1946) apud Fernandes et al. ${ }^{20}$

\section{Análise Estatística}

Os resultados foram avaliados no programa GraphPad Prism 5.0 (GraphPad Software, San Diego, CA, EUA). O teste de Shapiro-Wilk foi usado para determinar a normalidade da distribuição de dados. As variáveis paramétricas entre os três grupos foram determinadas pelo teste de análise de variância (ANOVA) com correção pelo pós-teste de Bonferroni. Foram consideradas diferenças estatisticamente significativas os valores de $p \leq 0,05$.

\section{Resultados}

\section{Índice Funcional do Ciático}

Na primeira avaliação, todos os valores do IFC foram semelhantes entre os grupos Sham + Nat $(-17,78)$, Enxerto $(-20,64)$ e Enx + Nat $(-14,184), \operatorname{com} p=0,6099$.

O grupo Sham + Nat obteve os índices nos limites da normalidade em todas as mensurações, permanecendo seus valores negativos e próximos do zero. Após o procedimento cirúrgico, os valores de IFC nos grupos experimentais (Enx e Enx + Nat) foram significativamente menores do que os do grupo Sham + Nat $(p<0,001$; - Tabela 1; - Figura 1). Nos grupos Enx e Enx + Nat, como o esperado, a $1^{\underline{a}}$ mensuração após a cirurgia de enxerto exibiu uma queda brusca nos valores do IFC30 ( $-145,9 \pm 26,06$ e $-89,40 \pm 7,501$, respectivamente).

No seguimento pós-operatório (60 e 90 dias), os 2 grupos enxertados tiveram uma melhora funcional progressiva, embora ainda inferiores à do grupo Sham + Nat $(p<0,0001)$, sem diferenças entre os grupos Enx e Enx + Nat. No IFC60, os grupos Enx e Enx + Nat apresentaram melhora nos valores de IFC $(-77,52 \pm 11,34$ e $-84,69 \pm 21,65$, respectivamente), e estes apresentaram valores semelhantes no IFC90 $(-72,14 \pm 19,50$ e $-84,27 \pm 5,78$, respectivamente).

Histologia da medula (contagem de motoneurônios) De acordo com o número de motoneurônios, podemos observar que houve diferença nas comparações entre os grupos Sham + Nat e Enxerto (647,1 $\pm 16,42$ versus $563,4 \pm 8,07$; $p<0,05)$ e entre os grupos Sham + Nat e EnX + Nat (647,1 $\pm 16,42$ versus $558,8 \pm 14,79 ; p<0,05)$, não havendo diferença entre os grupos Enxerto e Enx + Nat (-Figura 2).

\section{Discussão}

A natação é um exercício de reabilitação para tratar a lesão nervosa. Porém, o nível eficaz de resistência e de duração do exercício ainda não é claro. ${ }^{11}$

A natação foi aplicada nos ratos 14 dias após o procedimento cirúrgico, pois a reinervação funcional dos músculos inicia $\sim 2$ ou 3 semanas após o esmagamento do nervo ciático em ratos. 0 excesso de trabalho do músculo antes deste período pode ser prejudicial à recuperação. ${ }^{16,21,22}$

Os estudos que avaliam a neurorrafia término-terminal sem enxerto geralmente utilizam protocolos de reabilitação durante 4 semanas do pós-operatório $21,23,24$ ou um período menor. ${ }^{25}$ Pela aplicação do enxerto demonstrar pior prognóstico e uma recuperação clínica mais lenta, nós adotamos um aumento do período de exposição dos animais à natação.

No presente estudo, não foi observada nenhuma diferença clínica medida através do IFC. Os valores obtidos, entre 0 e -20, no período pré-operatório, refletiam uma função normal. ${ }^{26}$ Em nosso estudo, os valores de IFC próximos a -100 no grupo enxerto aos 60 dias apresentaram comprometimento completo da função nervosa, sugerindo ausência de

Tabela 1 Análise do índice funcional do ciático entre os grupos Sham + Natação, Enxerto e Enxerto + Natação nos tempos de 0 , 30,60 e 90 dias

\begin{tabular}{|l|l|l|l|l|l|l|l|l|}
\hline Tempo & Grupo & Média & DP & Mediana & Mínimo & Máximo & $n$ & valor-p \\
\hline $\mathbf{0}$ & Sham + Natação & $-17,78$ & 17,27 & $-24,27$ & $-25,74$ & 24,82 & 8 & 0,6099 \\
\hline & Enxerto & $-20,64$ & 9,679 & $-18,82$ & $-35,16$ & $-8,360$ & 9 & \\
\hline & Enxerto + Natação & $-14,18$ & 13,46 & $-17,83$ & $-25,57$ & $-25,57$ & 9 & \\
\hline $\mathbf{3 0}$ & Sham + Natação & $-34,64$ & 13,89 & $-32,41$ & $-50,20$ & $-13,79$ & 7 & $p<0,0001^{*}$ \\
\hline & Enxerto & $-145,9$ & 26,06 & $-143,3$ & $-190,2$ & $-100,4$ & 10 & \\
\hline & Enxerto + Natação & $-89,40$ & 7,501 & $-89,03$ & $-100,2$ & $-74,30$ & 9 & \\
\hline $\mathbf{6 0}$ & Sham + Natação & $-26,97$ & 13,28 & $-20,28$ & $-50,12$ & $-13,32$ & 7 & $p<0,0001^{\#}$ \\
\hline & Enxerto & $-77,52$ & 11,34 & $-80,24$ & $-91,15$ & $-58,36$ & 10 & \\
\hline & Enxerto + Natação & $-84,69$ & 21,65 & $-91,44$ & $-102,6$ & $-27,64$ & 10 & \\
\hline $\mathbf{9 0}$ & Sham + Natação & $-19,11$ & 11,33 & $-21,26$ & $-36,82$ & $-2,870$ & 8 & $p<0,0001^{\#}$ \\
\hline & Enxerto & $-72,14$ & 19,50 & $-73,37$ & $-107,5$ & $-41,16$ & 10 & \\
\hline & Enxerto + Natação & $-84,27$ & 5,782 & $-83,74$ & $-92,28$ & $-75,10$ & 8 & \\
\hline
\end{tabular}

Abreviação: DP, desvio padrão.

Nota: $n=$ número amostral. Teste de análise da variância (ANOVA) com correção pelo pós-teste de Bonferroni. Foram consideradas diferenças estatisticamente significativas valores $-\mathrm{p} \leq 0,05$. *; diferenças entre todas as comparações (Sham + Natação versus Enxerto, Sham + Natação versus Enxerto + Natação, Enxerto versus Enxerto + Natação); * ; diferenças entre os grupos: Sham + Natação versus Enxerto, Sham + Natação versus Enxerto + Natação. 


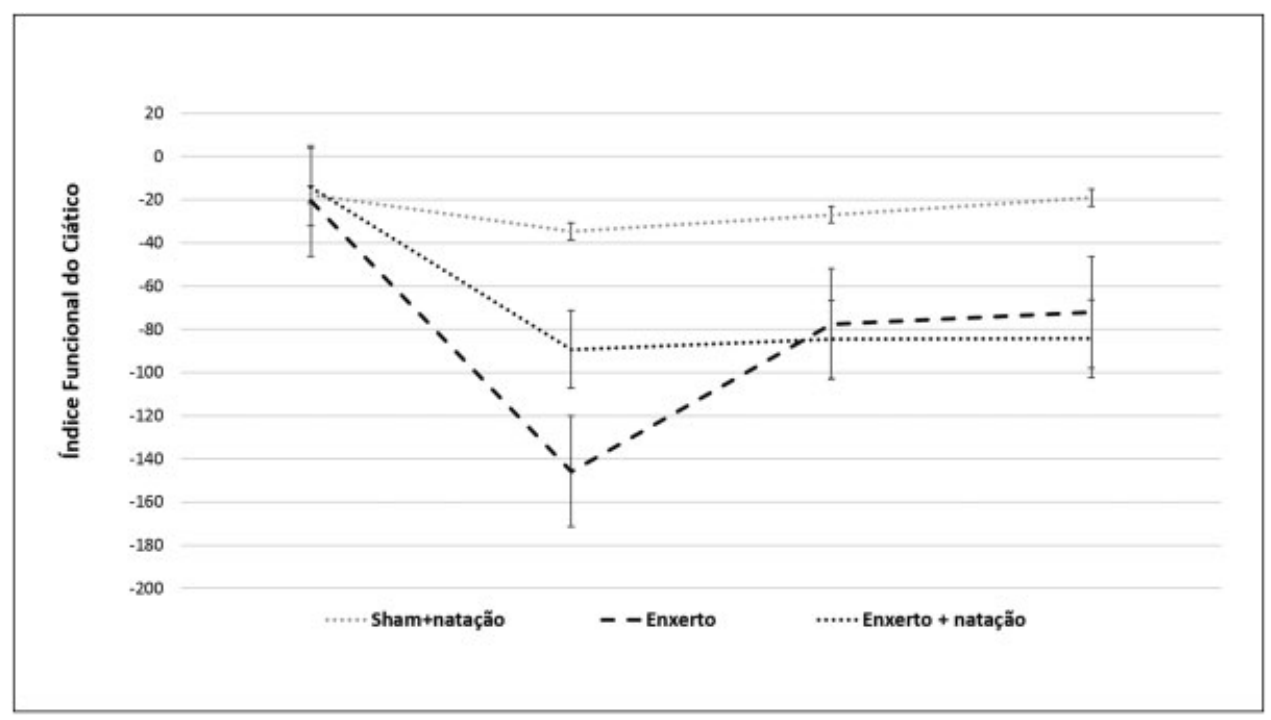

Fig. 1 Análise do índice funcional do ciático entre os grupos Sham + Natação, Enxerto e Enxerto + Natação nos tempos de 0, 30, 60 e 90 dias.

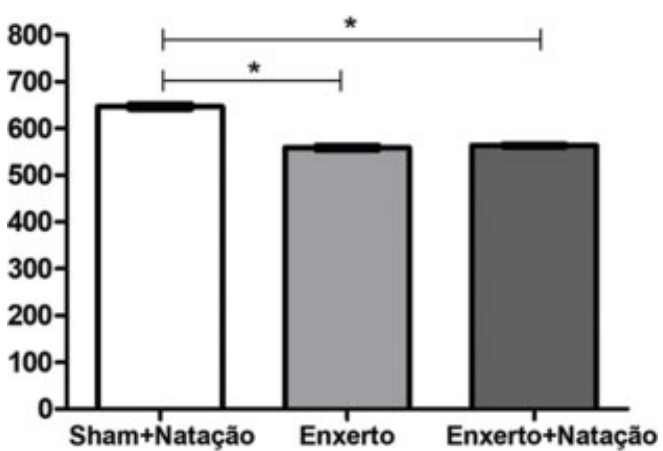

Fig. 2 Valores da contagem de motoneurônios após correção pelo fator Abercrombie. Foram avaliados 30 animais divididos nos seguintes grupos: Sham + Natação $(n=10)$, Enxerto $(n=10)$, e Enxerto + Natação $(n=10)$, nos tempos de $0,30,60$ e 90 dias. Teste de análise da variância (ANOVA) com correção pelo pós-teste de Bonferroni. * Foram encontradas diferenças entre as comparações (Sham + Natação versus Enxerto, Sham + Natação versus Enxerto + Natação), foram consideradas diferenças estatisticamente significativas valores-p $\leq 0,05$.

inervação nesse período. Nas demais avaliações, os valores dos grupos Enxerto e Enxerto + Natação se tornaram menos negativos, indicando retorno gradual da função. ${ }^{15} \mathrm{O}$ grupo Enxerto e Enx + Nat apresentaram um IFC menos negativo em 90 dias após o pós-operatório, mas este se distanciou dos valores apresentados pelo grupo controle.

Ilha et $\mathrm{al}^{21}$ observaram uma melhora precoce dos valores do IFC em animais submetidos ao exercício físico pós-cirurgia de esmagamento do nervo ciático quando comparados aos ratos sedentários.

Teodori et $\mathrm{al}^{9}$ avaliaram os aspectos morfológicos e as características funcionais dos nervos ciáticos de ratos e relataram que os animais submetidos à natação imediatamente após a lesão do nervo por esmagamento e animais submetidos à natação após 14 dias da lesão apresentaram menor número de axônios e maior diâmetro dos axônios e das fibras nervosas que animais controles, sugerindo que o exercício pode ser iniciado imediatamente após a lesão ou na fase tardia da lesão nervosa.

O número de motoneurônios marcados com Fluoro-Gold pode ser utilizado para aferir a reconexão dos nervos periféricos na medula espinhal. ${ }^{27}$ Quando avaliamos o número de motoneurônios, os valores do corno anterior da medula nos grupos Enxerto e Enx + Nat não mostraram nenhuma diferença estatística, permanecendo abaixo dos do grupo controle.

Tem sido relatado que animais submetidos à natação apresentaram regeneração nervosa acelerada em comparação a animais de controle com lesão nervosa do tipo esmagamento e o aumento do diâmetro das fibras nervosas. ${ }^{8}$

À semelhança dos estudos de Teodori et al. ${ }^{9}$ e Oliveira et al. ${ }^{15}$, o protocolo adotado aqui estabeleceu uma fase de adaptação dos animais à atividade de natação, que começa 20 minutos no primeiro dia e aumenta 10 minutos todos os dias até atingir 60 minutos no quinto dia. Esse período de adaptação permite que os animais se familiarizem com a natação, evitando tanto a acomodação física quanto o estresse. Por outro lado, existem algumas diferenças entre o protocolo utilizado no presente estudo e os demais dados presentes na literatura.

O método cirúrgico aqui adotado foi do tipo enxerto autólogo do nervo ciático, enquanto outros estudos utilizaram o esmagamento do nervo ciático ${ }^{9,16}$ e a transecção do nervo ciático. ${ }^{11} \mathrm{O}$ momento do início da intervenção aplicada foi no $14^{\circ}$ dia após o procedimento cirúrgico, e este período foi semelhante ao do estudo de Teodori et $\mathrm{al}^{9}$ e superior a outros protocolos que iniciaram a atividade após o $1^{\circ}$ dia $^{9,15}$ e o $7^{\circ}$ dia ${ }^{11}$ do método cirúrgico. Além disso, a duração da intervenção da natação foi superior aos estudos que aplicaram a atividade durante $2,{ }^{9} 3,{ }^{15}$ e 4 semanas. ${ }^{11}$ Estas variáveis podem ter afetado a eficácia da atividade da natação na regeneração do nervo ciático.

Dessa forma, a comparação entre os resultados obtidos e os achados da literatura é uma medida complexa, pois não 
existe homogeneidade quanto à metodologia aplicada, à técnica cirúrgica, ao tempo de início do protocolo, à intensidade, à frequência, e aos métodos de aferição.

\section{Conclusões}

Nosso estudo demonstrou que os animais submetidos ao protocolo de natação após o procedimento de enxerto do nervo ciático não apresentaram diferenças nos valores de IFC e nos números de motoneurônios quando comparados ao grupo controle. Concluímos que este tipo de protocolo descrito não é eficiente para reabilitação de lesões nervosas periféricas que necessitam de enxerto. Novos estudos são necessários para avaliar métodos que acelerem e melhorem os resultados funcionais após o procedimento de enxerto do nervo ciático.

Estudo realizado com animais. Segue aprovação (CEUA n ${ }^{\circ}$ 5060051217).

\section{Conflict of Interests}

The authors have no conflict of interests to declare.

\section{Referências}

1 Bonetti LV, Malysz T, Ilha J, Barbosa S, Achaval M, Faccioni-Heuser MC. The Effects of Two Different Exercise Programs on the Ultrastructural Features of the Sciatic Nerve and Soleus Muscle After Sciatic Crush. Anat Rec (Hoboken) 2017;300(09):1654-1661

2 Torres RY, Miranda GE. Epidemiology of Traumatic Peripheral Nerve Injuries Evaluated by Electrodiagnostic Studies in a Tertiary Care Hospital Clinic. Bol Asoc Med P R 2015;107(03):79-84

3 Caierao QM, Betini J, Teodori RM, Minamoto VB. The effect of time interval between electrical stimulation on the denervated rat muscle. Rev Bras Fisioter 2008;12(02):143-148

4 Malanotte JA, Kakihata CMM, Karvat J, Brancalhão RMC, Ribeiro LFC, Bertolini GRF. Jumping in aquatic environment after sciatic nerve compression: nociceptive evaluation and morphological characteristics of the soleus muscle of Wistar rats. Einstein (Sao Paulo) 2017;15(01):77-84

5 Wong KH, Naidu M, David P, et al. Peripheral nerve regeneration following crush injury to rat peroneal nerve by aqueous extract of medicinal mushroom Hericium erinaceus (Bull.: Fr) Pers. (Aphyllophoromycetideae). Evid Based Complement Alternat Med 2011; 2011:580752

6 Medeiros A, Oliveira EM, Gianolla R, Casarini DE, Negrão CE, Brum PC. Swimming training increases cardiac vagal activity and induces cardiac hypertrophy in rats. Braz J Med Biol Res 2004;37(12): 1909-1917

7 Sarikcioglu L, Oguz N. Exercise training and axonal regeneration after sciatic nerve injury. Int J Neurosci 2001;109(3-4):173-177

8 Doyle LMF, Roberts BL. Exercise enhances axonal growth and functional recovery in the regenerating spinal cord. Neuroscience 2006;141(01):321-327

9 Teodori RM, Betini J, de Oliveira LS, Sobral LL, Takeda SY, de Lima Montebelo MI. Swimming exercise in the acute or late phase after sciatic nerve crush accelerates nerve regeneration. Neural Plast 2011;2011:783901
10 Kavlak E, Belge F, Ünsal C, Üner AG, Cavlak U, Cömlekçi S. Effects of pulsed electromagnetic field and swimming exercise on rats with experimental sciatic nerve injury. J Phys Ther Sci 2014;26(09): 1355-1361

11 Liao CF, Yang TY, Chen YH, Yao CH, Way TD, Chen YS. Effects of swimming exercise on nerve regeneration in a rat sciatic nerve transection model. Biomedicine (Taipei) 2017;7(01):3

12 Rocha JC. Hidroginástica. Teoria e prática. Rio de Janeiro: Sprint; 1994

13 Ruoti RG, Morris DM, Cole AJ. Reabilitação Aquática. São Paulo: Manole; 2000

14 van Meeteren NL, Brakkee JH, Helders PJGW, Gispen WH. The effect of exercise training on functional recovery after sciatic nerve crush in the rat. J Peripher Nerv Syst 1998;3(04):277-282

15 Oliveira LS, Sobral LL, Takeda SY, et al. Estimulación eléctrica y natación en la fase aguda de la axonotmesis: influencia sobre la regeneración nerviosa y la recuperación funcional. Rev Neurol 2008;47(01):11-15

16 Herbison GJ, Jaweed MM, Ditunno JF. Effect of swimming on reinnervation of rat skeletal muscle. J Neurol Neurosurg Psychiatry 1974;37(11):1247-1251

17 van Meeteren NL, Brakkee JH, Hamers FP, Helders PJ, Gispen WH. Exercise training improves functional recovery and motor nerve conduction velocity after sciatic nerve crush lesion in the rat. Arch Phys Med Rehabil 1997;78(01):70-77

18 Fernandes M, Valente SG, Fernandes MJ, et al. Bone marrow cells are able to increase vessels number during repair of sciatic nerve lesion. J Neurosci Methods 2008;170(01):16-24

19 Roth F, Fernandes M, Valente SG, et al. Platelet-rich fibrin conduits as an alternative to nerve autografts for peripheral nerve repair. J Reconstr Microsurg 2017;33(08):549-556

20 Fernandes M, Valente SG, Amado D, et al. Estudo comparativo entre enxerto autógeno e enxerto muscular coberto com tubo de veia autógeno em nervos tibiais de ratos Wistar, utilizando o fluoro-gold ${ }^{\circledR}$ como marcador neuronal. Acta Ortop Bras 2007;15 (02):97-100

21 Ilha J, Araujo RT, Malysz T, et al. Endurance and resistance exercise training programs elicit specific effects on sciatic nerve regeneration after experimental traumatic lesion in rats. Neurorehabil Neural Repair 2008;22(04):355-366

22 Sobral LL, Oliveira LS, Takeda SY, et al. Immediate versus late exercise for rat sciatic nerve regeneration after axonotmesis: histomorphometric and functional analysis. Rev Bras Fisioter 2008;12(04):311-316

23 Asensio-Pinilla E, Udina E, Jaramillo J, Navarro X. Electrical stimulation combined with exercise increase axonal regeneration after peripheral nerve injury. Exp Neurol 2009;219(01):258-265

24 Jang SH, Lee JH. Effects of physical exercise on the functional recovery of rat hindlimbs with impairments of the sciatic nerve as assessed by 2D video analysis. J Phys Ther Sci 2015;27(03):935-938

25 Krakowiak J, Liu C, Papudesu C, Ward PJ, Wilhelm JC, English AW. Neuronal BDNF signaling is necessary for the effects of treadmill exercise on synaptic stripping of axotomized motoneurons. Neural Plast 2015;2015:392591

26 Dash H, Kononov A, Prayson RA, Petras S, Browne EZ. Evaluation of nerve recovery from minimal-duration crush injury. Ann Plast Surg 1996;37(05):526-531

27 Dellon AL, Mackinnon SE. Sciatic nerve regeneration in the rat. Validity of walking track assessment in the presence of chronic contractures. Microsurgery 1989;10(03):220-225 